\title{
Morphological Evolution of Electrochemically Plated/Stripped Lithium Microstructures Investigated by Synchrotron X-Ray Phase Contrast Tomography**
}

\author{
Fu Sun, ${ }^{* \dagger, \S}$ Lukas Zielke, ${ }^{\ddagger}$ Henning Markötter, ${ }^{\dagger, \S}$ André Hilger, ${ }^{\dagger, \S}$ Dong Zhou, ${ }^{\dagger, \S}$ Riko Moroni, ${ }^{\dagger}$ \\ Roland Zengerle, ${ }^{\ddagger}$ Simon Thiele, ${ }^{\ddagger, \#}$ John Banhart ${ }^{\dagger, \S}$ and Ingo Manke ${ }^{\S}$ \\ ${ }^{\dagger}$ Institute of Material Science and Technologies \\ Technical University Berlin \\ Strasse des 17. Juni 135, 10623 Berlin, Germany \\ ${ }^{\ddagger}$ Laboratory for MEMS Applications, IMTEK Department of Microsystems Engineering \\ University of Freiburg \\ Georges-Koehler-Allee 103, 79110 Freiburg, Germany \\ E-mail: Lukas.Zielke@imtek.de \\ ${ }^{\S}$ Helmholtz Centre Berlin for Materials and Energy \\ Hahn-Meitner-Platz 1, \\ 14109 Berlin, Germany \\ ${ }^{\text {\#}}$ FIT, University of Freiburg \\ Georges-Köhler-Allee 105, 79110 Freiburg, Germany, \\ *Email: fu.sun@helmholtz-berlin.de \\ **ACS Nano 10(8), 7990- 7997 (2016) - [R] \\ American Chemical Society, ISSN: 1936-0851 / 1936-086X — doi: 10.1021/acsnano.6b03939
}

\begin{abstract}
Due to its low redox potential and high theoretical specific capacity Li metal has drawn worldwide research attentions because of its potential use in next-generation battery technologies such as $\mathrm{Li}-\mathrm{S}$ and $\mathrm{Li}-\mathrm{O}_{2}$. Unfortunately, uncontrollable growth of $\mathrm{Li}$ microstructures (LmSs, e.g. dendrites, fibers) during electrochemical $\mathrm{Li}$ stripping/plating has prevented their practical commercialization. Despite various strategies proposed to mitigate LmS nucleation and/or block its growth, a fundamental understanding of the underlying evolution mechanisms remains elusive. Herein, synchrotron in-line phase contrast X-ray tomography was employed to investigate the morphological evolution of electrochemically deposited/dissolved LmSs non-destructively. We present a 3D characterization of electrochemically stripped Li electrodes with regard to electrochemically plated LmSs. We clarify fundamentally, the origin of the porous lithium interface growing into Li electrodes. Moreover, cleavage of the separator caused by growing LmS was experimentally observed and visualized in 3D. Our systematic investigation provides fundamental insights into LmS evolution and enables us to understand the evolution mechanisms in Li electrodes more profoundly.
\end{abstract}

The further development of characterization methods has often promoted a better understanding of the structure and function of materials. ${ }^{1-6}$ For example, optical microscopy, ${ }^{7}$ atomic force microscopy (AFM), ${ }^{8}$ scanning electron microscope (SEM), ${ }^{9}$ transmission electron microscopy (TEM) ${ }^{10}$ nuclear magnetic resonance (NMR) ${ }^{11}$ and magnetic resonance imaging (MRI) ${ }^{12}$ have been employed to understand the growth of the lithium microstructure (LmS) in lithium-ion batteries. Our comprehension of the growth mechanisms of LmSs has greatly improved $^{13,14}$ since usage of Li metal as negative electrode began in the 1970s. ${ }^{15}$ Nevertheless, prior analytical tools are inherently limited. For instance, most techniques usually require prior sample removal from their as grown environment for analysis, which is problematic since accidental exposure of LmSs to air during sample transfer ${ }^{16}$ and the Lirinsing procedures applied for sample preparation ${ }^{17}$ may fundamentally change their morphology. Furthermore, nuclear resonance techniques suffer from limited resolution of around $100 \mu \mathrm{m}$, the limited penetration depth of radiofrequency irradiation and additional 
concerns arising from artefacts introduced by metals. ${ }^{18}$ Recently, Eastwood et al. have characterized the 3D microstructure of electrodeposited LmS by synchrotron in-line phase contrast X-ray tomography at sub- $\mu \mathrm{m}$ resolution, ${ }^{18}$ demonstrating that this method is a suitable and powerful tool for the study of LmSs. In another study, Harry et al. found that most Li dendrites reside within the electrode instead of the polymer separator during the early stage of dendrite development. They conclude that these dendrites preferentially grow from crystalline impurities existing within an electrode. ${ }^{19}$

In this work, we implement a further systematic study by using in-line phase contrast X-ray tomography. We propose that the Li dendrites residing on the surface of the electrode arise from the nascent electrochemically deposited LmS occupying the previously dissolved initial Li bulk electrode. Furthermore, the present study clarifies, from a fundamental point of view, the origin of the Porous Lithium Interface (PLI) growing towards the Li electrode, which has been discovered previously. ${ }^{20}$ In addition, an unexpected cleavage of the separator caused by the growing LmS is experimentally shown. These unprecedented findings, which were not accessible from conventional microscopic characterizations and electrochemical measurements, fundamentally deepen our understanding of the evolution mechanism of the LmS during electrochemical stripping/plating. The insights obtained could open up the way towards design principles and opportunities to further ameliorate or eliminate LmSs.

\section{RESULTS AND DISCUSSION}

A proof-of-concept electrochemical cell, which is fully compatible with X-ray imaging and at the same time representative for commercial lithium-ion batteries (LIBs), was designed and manufactured as shown in Figure 1 along with a schematic illustration of the synchrotron Xray imaging setup at BAMline of BESSY II, Berlin, Germany. ${ }^{21}{ }^{22}$ Two kinds of electrochemical cells were investigated: a Li electrode coupled with a silicon-based composite (Si/C) electrode (Li-Si half-cell) and a $\mathrm{Li}$ electrode coupled with a $\mathrm{Li}$ electrode (Li- $\mathrm{Li}$ symmetrical cell). All cells employed commercial Celgard ${ }^{\circledR} 2325$ separators and were filled with $0.2 \mathrm{ml}$ standard $1 \mathrm{M} \mathrm{LiPF}_{6}$ in ethylene carbonate (EC) and ethyl methyl carbonate (EMC) $(\mathrm{EC} / \mathrm{EMC}=50 / 50(\mathrm{v} / \mathrm{v}))$ electrolyte. Details of parameters and cycling routines of the investigated cells are given in the Methods section and the Supporting Information (SI). The validation of the electrochemical performance of this proof-of-concept cell can be found in previous reports. ${ }^{23,24}$ The cycling curves of all the cells are shown in the SI. After cycling, the cells were transferred to the beamline to conduct tomography without prior disassembly. Every tomography was recorded with 2200 projections covering a $180^{\circ}$ rotation angle. A detector system with $0.438 \mu \mathrm{m}$ pixel size was used. ${ }^{25}, 26$ The field of view (FoV) is $1.7 \times 1.2$ $\mathrm{mm}^{2}$ large (length $\times$ height), which enables the simultaneous imaging of morphological changes occurring in all components of the electrodes. The detailed normalization, tomography reconstruction and 3D presentation procedure is given in the Methods section and in SI. ${ }^{27}$
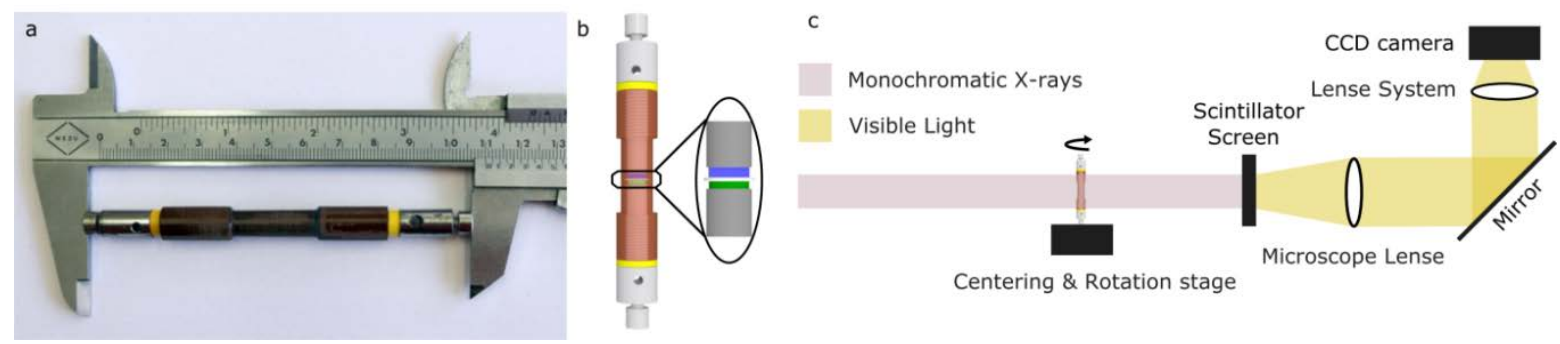

Figure 1 Schematic illustration of the proof-of-concept cell and the beamline setup. a, photograph and b, corresponding illustration of the electrochemical cell: polyamide-imide housing (brown), two screws serving as current collectors (light gray), two sealing rings (yellow), cathode (blue), separator (white) and anode (green). c, schematic representation of the experimental setup at BAMline BESSY II, Berlin, Germany. More information about the cell and the setup parameters can be found in Methods and SI. 
Cross-sectional views of uncycled $\mathrm{Li}-\mathrm{Li}$ (Li-Li-N: N denotes sample cell number. In this case, the sample is Li-Li-1) and Li-Si (Li-Si-N: N denotes sample cell number. In this case, the sample is Li-Si-1) cells are shown in Figure 2a-c and Figure 3a-c, respectively, where we can clearly discern the $\mathrm{Li}$ electrode, the separator and the counter $\mathrm{Li}$ electrode ( $\mathrm{Si} / \mathrm{C}$ electrode) by the light and dark interfaces between them arising from the in-line phase contrast. ${ }^{28}$ In both cases, the interfaces between electrode and electrolyte of uncycled cells exhibit a few minor noticeable features (uneven surfaces shown in Figure 2c and Figure 3c) caused by the high electrochemical activity of $\mathrm{Li}^{19}$ The negative electrode and positive electrode are labeled separately in Figure 2 and Figure 3 (the arrangement is taken only for easy demonstration: in sample Li-Li-2 (Figure 2d-l), the Li negative electrode is the bottom electrode, while the $\mathrm{Li}$ positive electrode is the upper. In sample Li-Li-3 (Figure $2 \mathrm{~m}-\mathrm{o}$ ), the Li negative electrode is the top, the $\mathrm{Li}$ positive electrode the bottom electrode. For all Li-Si half cells, the $\mathrm{Li}$ anode is the top, the Si/C cathode the bottom electrode (Figure 3)). Figure 2d,e show images of the LiLi-2 sample subjected to a constant current of $0.3 \mathrm{~mA} \cdot \mathrm{cm}^{-2}$ for $10 \mathrm{~h}$. The corresponding 3D representation is shown in Figure 2f. Compared to Figure 2a-c, the initially flat electrode/electrolyte interfaces have turned into rugged interfaces with numerous electrochemically deposited LmSs on the positive side. In addition, differently sized cavities can be observed in the partially electrochemically dissolved $\mathrm{Li}$ negative electrode. It has been reported that the appearance of such cavities stems from the unevenly formed solid electrolyte interface (SEI) and that regions containing cavities are electrochemically more active than flat surfaces. ${ }^{29}$ Furthermore, the separator deforms into an arched shape and adheres closely to the electrochemically dissolved porous structure, indicating that significant stress is generated during LmS formation.

In a next step, the Li-Li-2 sample was continuously electrochemically stripped for another $19 \mathrm{~h}$. Figure 2g-I illustrates that a large amount of $\mathrm{Li}$ is further dissolved from the Li negative electrode and deposited onto the Li positive electrode, as indicated by the increased volume of cavities within the negative electrode and by the increasing LmSs on top of the positive electrode. Consequently, some delamination between the separator and the two Li electrodes can be observed, which is comparable to previous reports. ${ }^{17}$

After electrochemically plating the Li-Li-2 sample for $17 \mathrm{~h}$, the structure shown in Figure $2 \mathrm{j}-\mathrm{l}$ is obtained. One observes that: i) during $\mathrm{Li}$ stripping from the positive electrode, it is the remaining Li bulk instead of the electrochemically plated LmS that undergoes electrochemical $\mathrm{Li}$ dissolution, as indicated by the newly formed void in the Li positive electrode (indicated by white triangles in Figure $2 \mathrm{j}, \mathrm{k}$ ), ii) during plating of the negative electrode with $\mathrm{Li}, \mathrm{Li}$ deposition occurs preferentially in the system of cavities structures as indicated by the newly formed LmSs on the surface of the existing cavities (note white tetragons in Figure 2j,k). It has been reported that the nascent electrochemically inactive LmSs are still chemically active and can easily react with the electrolyte to form a new SEI coverage. ${ }^{20}$ Consequently, the SEI formed electrically insulates most of the nascent LmSs, thereby deactivating them electrochemically during Li stripping. ${ }^{30}$ The reason for the preferential deposition of LmSs in cavity-containing regions is that such areas are electrochemically more active ${ }^{31}$ or possess a high local ionic conductivity. ${ }^{29}$

Figure 2m-o shows a cross section of the Li-Li-3 sample which has been electrochemically stripped at a constant current of $0.3 \mathrm{~mA} \cdot \mathrm{cm}^{-2}$ for $30 \mathrm{~h}$. Here, the unexpected phenomenon of cleavage of the separator due to the puncture by the LmS is observed. The corresponding 3D representation is displayed in Figure 2o. The presence of $\mathrm{Li}$ filaments running across the separator is observed, as indicated by white arrows between the punctured separator in Figure $2 \mathrm{~m}$. The cleavage can be attributed to the anisotropic mechanical properties of the Celgard ${ }^{\circledR}$ separator in the transverse direction due to the manufacturing process employed to create the 
micro-porous membranes. ${ }^{32}$ This is the first time that the puncture of a separator is reported experimentally and displayed in 3D. 3D representations of the morphological evolution of these cells are shown in Figure 2c, f, i, l, o. The locations of the cross sections shown in Figure 2, along with the corresponding videos displaying the 3D nature of LmSs are available in SI.
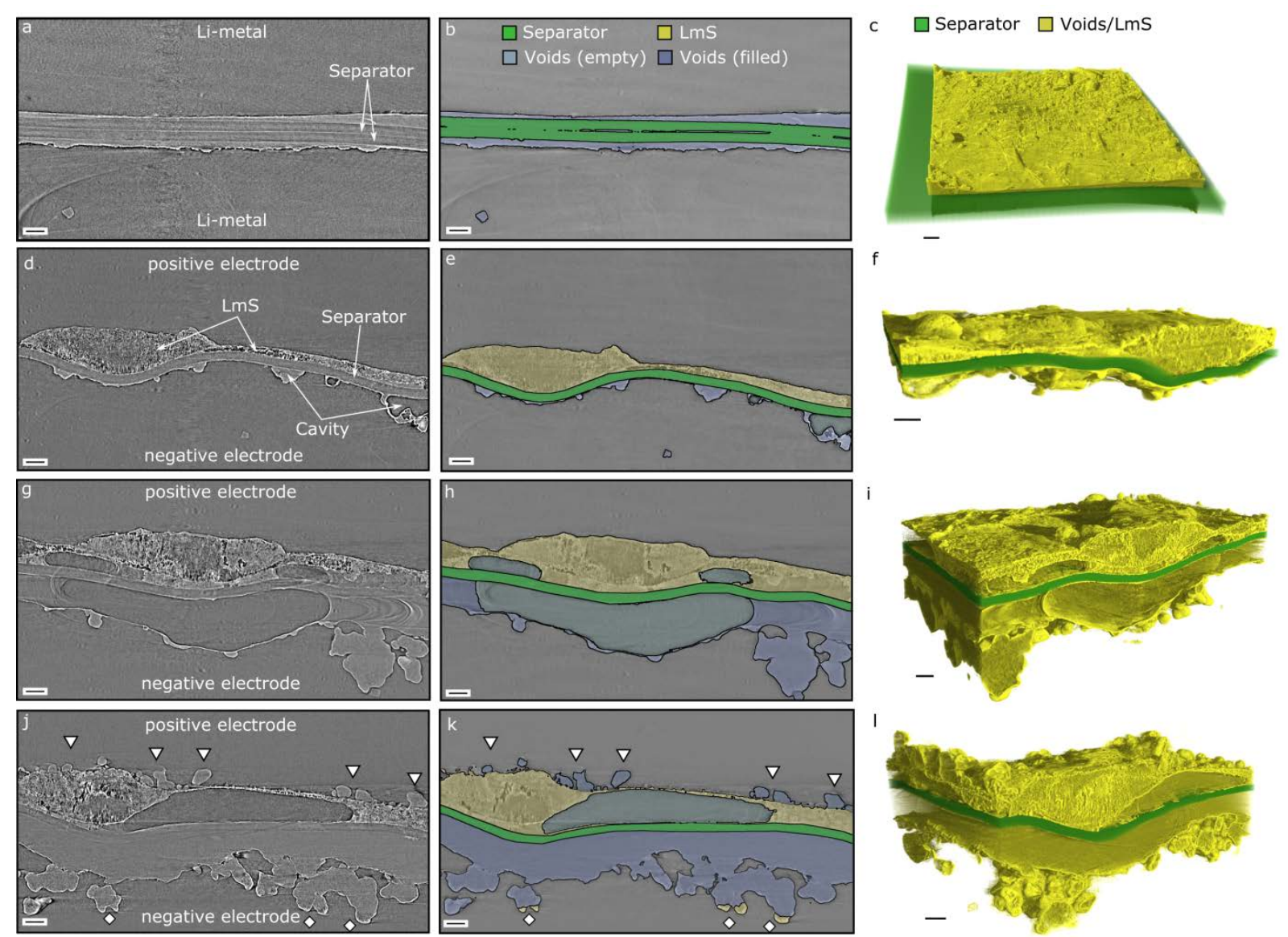

$-$
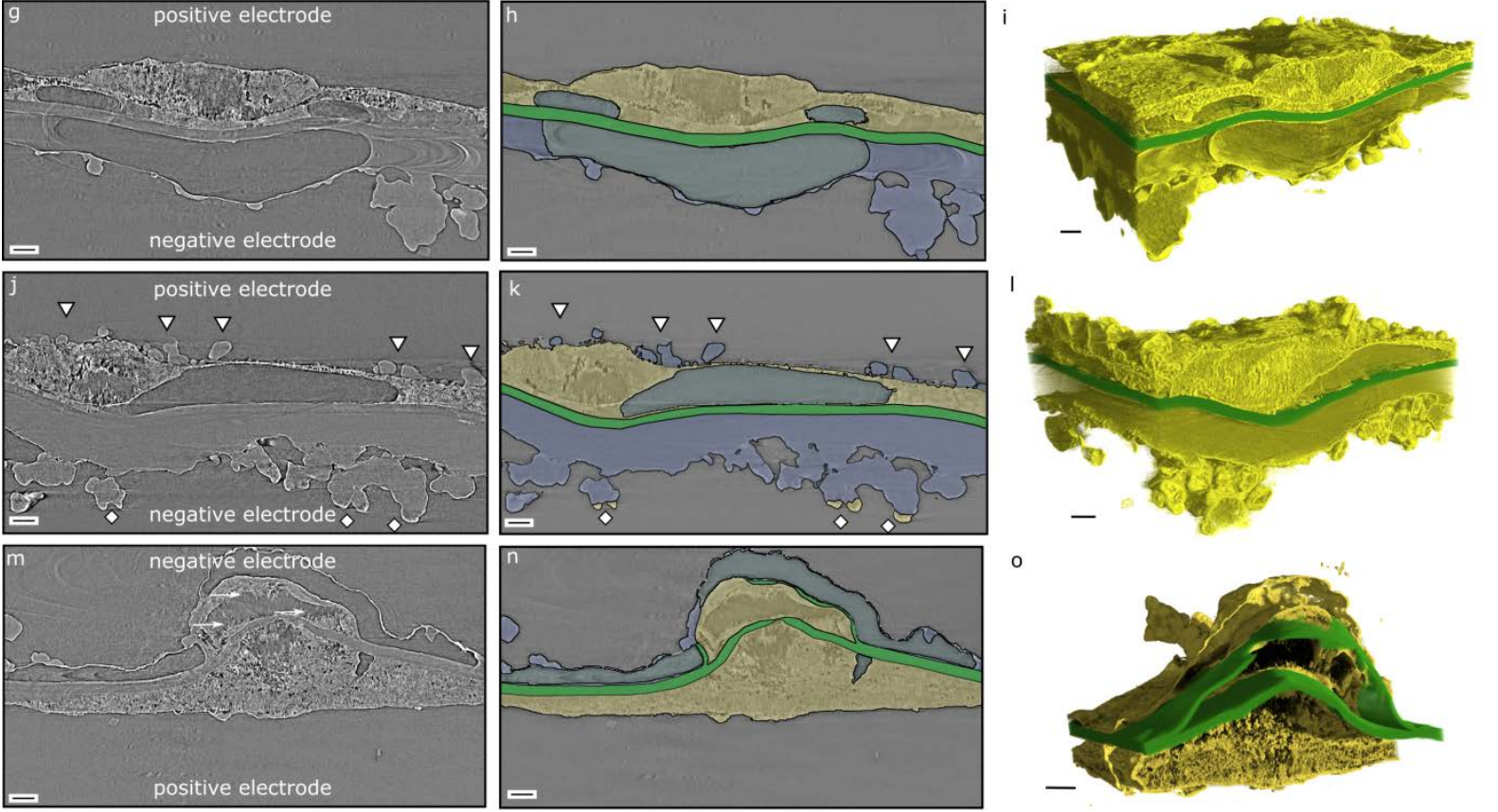

Figure 2 Morphological evolution of LmS in Li-Li symmetrical cells. a-c, uncycled pristine Li-Li-1 sample. The Celgard ${ }^{\circledR}$ separator is sandwiched between two Li electrodes. d-f, Li-Li-2 sample after electrochemically stripping for $10 \mathrm{~h}$ at $0.3 \mathrm{~mA} \cdot \mathrm{cm}^{-2}$. Li metal has been dissolved from the Li negative electrode (evidenced by the visible cavities) and deposited onto the Li positive electrode (evidenced by the LmS). g-i, Li-Li-2 sample after electrochemically stripping for another $19 \mathrm{~h}$ at $0.3 \mathrm{~mA} \cdot \mathrm{cm}^{-2}$. An increasing amount of $\mathrm{Li}$ has been dissolved from the $\mathrm{Li}$ negative electrode and deposited onto the $\mathrm{Li}$ positive electrode. $\mathrm{j}-\mathrm{l}$, Li-Li-2 cell after electrochemically plating for $19 \mathrm{~h}$ at $0.3 \mathrm{~mA} \cdot \mathrm{cm}^{-2}$ showing that the residual Li bulk (indicated by white triangles) instead of LmSs has been dissolved and preferentially deposited onto the surface of existing cavities (marked by white tetragons). m-o, Li-Li-3 sample electrochemically stripping for $30 \mathrm{~h}$ at $0.3 \mathrm{~mA} \cdot \mathrm{cm}^{-2}$ showing filamentous LmSs running across the separator (indicated by arrows) and the torn Celgard ${ }^{\circledR}$ separator. The first column (a, d, g, j, m) contains reconstructed X-ray tomographies. The second column (b, e, h, k, n) shows the same slice after a combination of manual and automated phase filtering and color labeling. The third column (c, f, i, l and o) shows the corresponding 3D representations. All the scale bars are $50 \mu \mathrm{m}$ long. The cycling procedures of all cells, the detailed phase filtering procedure and 3D presentation methods are described in Methods and SI. 
More information about the evolution of LmSs can be obtained from tomographies showing the Li-Si half-cells. Figure 3d-f displays cross-sectional views of the Li-Si-2 sample that was galvanostatically discharged for $15 \mathrm{~h}$ at $0.26 \mathrm{~mA} \cdot \mathrm{cm}^{-2}$. Compared to the uncycled Li electrode sample (Figure 3a-c), a good deal of Li has been electrochemically stripped from the bulk anode $\mathrm{Li}$ (evidenced by the cavities formed as marked by white triangles in Figure 3d,e) and subsequently alloyed with Si particles (evidenced by the cracks formed as marked by white diamonds in Figure 3d,e). Moreover, after the Li-Si-1 sample has been discharged for $15 \mathrm{~h}$ and then charged for $5 \mathrm{~h}$ at $0.7 \mathrm{~mA} \cdot \mathrm{cm}^{-2}$, some but not all voids generated during electrochemical dissolution are dominated by the nascent electrochemically deposited LmSs, as shown in Figure 3g-i. This non-uniform filling of voids may be the result of the uneven distribution of Li ion flux. ${ }^{14}$ Finally, the Li-Si-3 sample, which has experienced 5 cycles, is shown in Figure 3j-l. We observe that a nearly $100-\mu \mathrm{m}$ thick portion of the initial Li anode has been dissolved and is thereafter occupied by electrochemically generated LmSs. On the Si cathode, almost all of the Si particles have been fully lithiated to $\operatorname{SiLi}_{\mathrm{x}}(1<\mathrm{x}<4)$ and are no longer observable due to the low X-ray attenuation coefficient. ${ }^{33} 3 \mathrm{D}$ representations of the morphological evolution of the LmS formed in Li-Si cells are shown in Figure 3c, f, i, l. The locations of the cross sections shown in Figure 3, along with the corresponding videos showing the 3D nature of the LmS are available in SI.
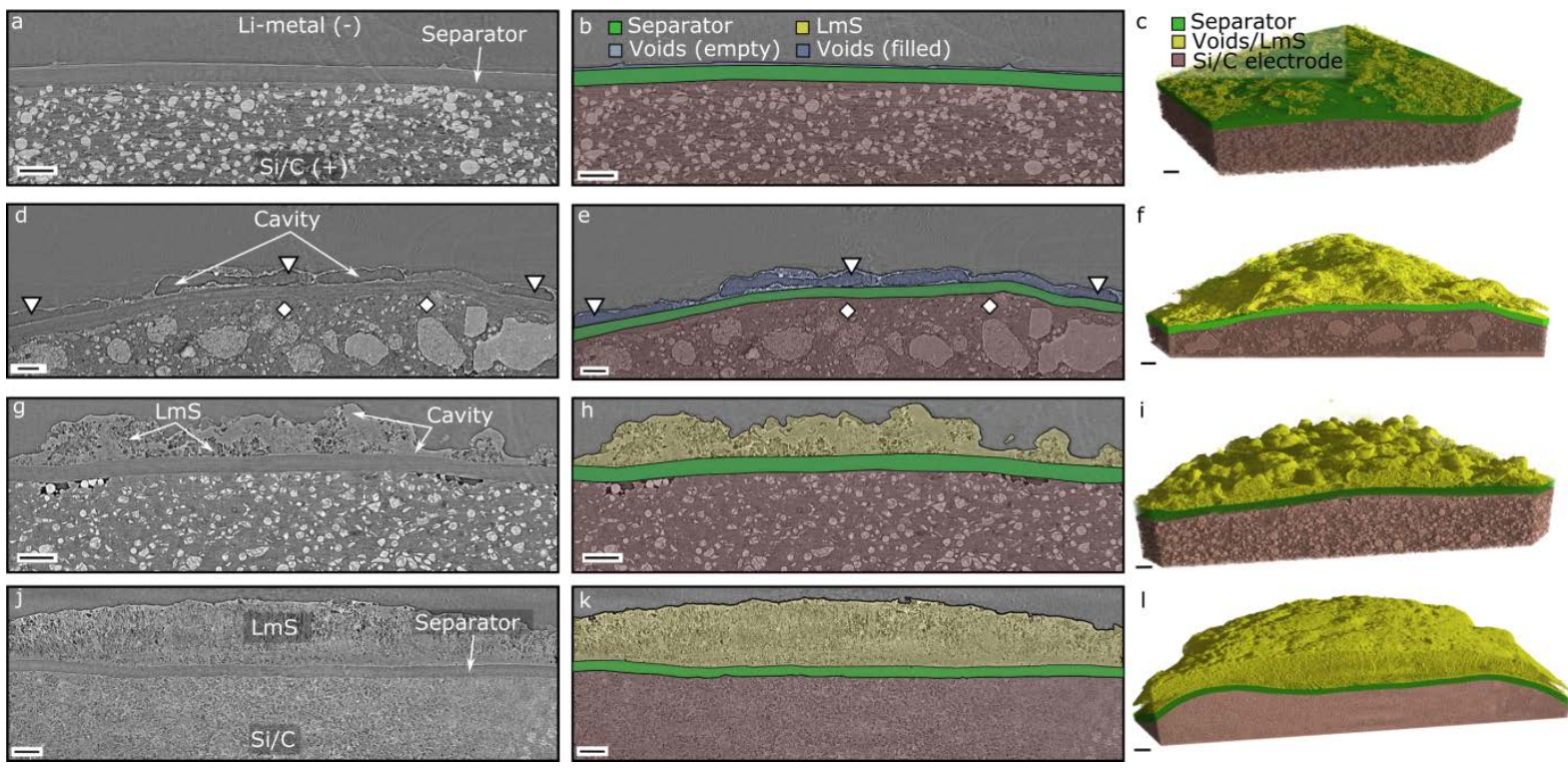

Figure 3 Morphological evolution of LmS in Li-Si half cells. a-c, uncycled pristine Li-Si-1 cell. The Celgard $^{\circledR}$ separator is sandwiched between the $\mathrm{Li}$ anode and the $\mathrm{Si} / \mathrm{C}$ composite cathode. d-f, Li-Si-2 sample that has been galvanostatically discharged for $15 \mathrm{~h}$ at $0.26 \mathrm{~mA} \cdot \mathrm{cm}^{-2}$. Here, Li has been dissolved from the $\mathrm{Li}$ anode (evidenced by cavity/void, white triangles) and alloyed with the $\mathrm{Si} / \mathrm{C}$ cathode (evidenced by Si crack formation, white tetragons). g-i, Li-Si-1 cell after $15 \mathrm{~h}$ of discharge and $5 \mathrm{~h}$ of charge at $0.7 \mathrm{~mA} \cdot \mathrm{cm}^{-2}$. The cavities formed are partially occupied by newly formed LmSs. j-l, Li-Si-3 cell that has experienced 5 cycles (see SI). Here, nearly half of the original Li anode has been dissolved and is now occupied by deposited LmSs. The first column (a, d, g, j) contains reconstructed tomography slices. The second column (b, e, h, k) shows the same slice after a combination of manual and automated phase filtering and color labeling. The third column (c, f, i, l) contains the corresponding 3D representations. All the scale bars are $50 \mu \mathrm{m}$ long. The cycling procedures of all cells can be found in SI.

Based on these systematic investigations, we suggest a model that describes the phenomena observed in Figures 2 and 3. A schematic illustration of the proposed evolution is shown in Figure 4. During the $1^{\text {st }}$ electrochemical Li stripping, a significant amount of $\mathrm{Li}$ dissolves from the Li anode (yellow), and numerous cavities (white) are generated. Meanwhile, these Li ions, i) are electrochemically deposited into the LmS on the hostless Li cathode (as observed in Figure 2) or, ii) form an alloy with the Si/C composite electrode (as observed in Figure 3). 
The purple-yellow color gradient in Figure 4 indicates the two cases observed in Figure 2 and Figure 3. During subsequent electrochemical Li plating, the Li ions are transported back to the $\mathrm{Li}$ anode and are preferentially deposited in the previously formed cavities due to the high electrochemical local activity there. However, during the following (the $2^{\text {nd }}$ ) Li stripping from the anode, it is the remaining bulk $\mathrm{Li}$ (yellow) that undergoes electrochemical dissolution instead of the nascent electrochemically deposited LmS (ocre, formed during previous Li plating) which is covered by electron-blocking SEI. Summarizing this process one can say that the initial bulk Li anode is electrochemically dissolved continuously in each Li stripping step, which results in numerous cavities. During subsequent Li plating, large amounts of the LmS are generated and form the PLI, which occupies the voids formed during previous Li dissolution. It has been reported that a continuously growing PLI can substantially increase the internal resistance of a cell, which eventually results in poor cell performance and ultimately in cell failure. ${ }^{20}$ Meanwhile, thin LmS protruding from the PLI towards the separator conducts high currents and consequently experiences large localized Joule heating. Previous simulations demonstrate that this localized Joule heating can be as high as $10^{8} \mathrm{~W} \mathrm{~m}^{-3}$ and it could result in the melting of the separator and finally puncture of the separator. ${ }^{34}$

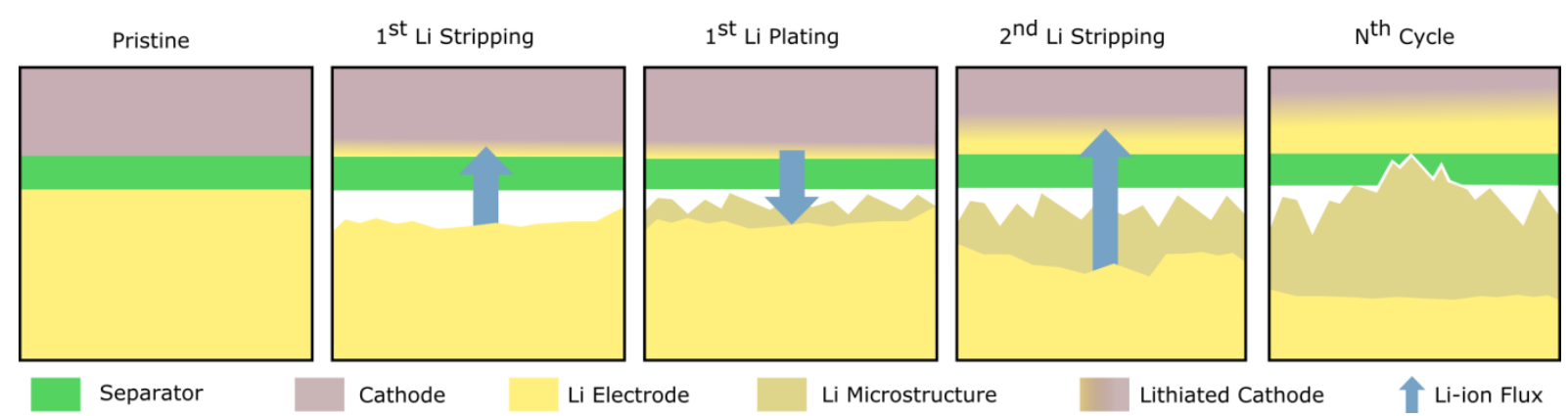

Figure 4 Schematic illustration of the morphological evolution of LmSs in a Li anode: from left to right, a pristine state cell; $\mathrm{Li}$ anode then undergoes the $1^{\text {st }}$ stripping during discharge; $\mathrm{Li}$ anode undergoing the $1^{\text {st }}$ plating during charge; Li anode undergoing the $2^{\text {nd }}$ stripping during another discharge; $\mathrm{Li}$ anode after $\mathrm{N}^{\text {th }}$ stripping/plating $(\mathrm{N} \geq 2)$.

The observations of the evolution of electrochemically stripped/plated LmSs presented here shed light on a range of processes that previously have not been acknowledged or barely considered. Firstly, the proposed mechanism of LmS evolution can explain the origin of the electrochemically generated PLI that grows into the $\mathrm{Li}$ bulk electrode. Based on our observations, both the electrochemical dissolution of the initial Li bulk electrodes and the following electrochemical deposition of nascent LmSs contribute to the inward growth of the PLI. Secondly, the evaluation of using Coulombic efficiency to investigate the cycling efficiency of $\mathrm{Li}$ in $\mathrm{Li}$ symmetrical cells is compromised by the electrochemically inert LmSs. ${ }^{29}$ According to our observations, a significant amount of $\mathrm{Li}$ is stripped from the initial $\mathrm{Li}$ bulk instead from the generated nascent (electrochemically inactive) LmSs to compensate for the depletion of Li ions in the electrolyte used to be plated. Thirdly, concerns about using $\mathrm{Li}$ metal as a standard counter electrode for the evaluation of the performance of battery materials using half-cells are raised. In fact, it has been confirmed that the PLI, which continuously grows in each cycle, can markedly increase the inner cell resistance and is the actual origin of the onset of cell degradation and failure. ${ }^{20}$ Finally, considering that the dissolution of the initial Li bulk electrodes and the deposition of nascent LmSs (necessitated by the charge transfer within cells) remain intrinsic and unavoidable, the present results suggest that fundamental strategies that involve direct engineering of $\mathrm{Li}$ electrodes are desirable and necessary. For example, strategies such as introducing surface patterns to the $\mathrm{Li}$ electrode to direct and control $\mathrm{Li}$ deposition, ${ }^{35}$ replacing conventional $\mathrm{Li}$ foil electrodes by organic-coated Li powder ${ }^{36}$ or employing different substrates to selectively deposit and then 
encapsulate the $\mathrm{LmS},{ }^{37}$ as well as adding suitable additives into the electrolyte to facilitate the formation of self-aligned $\mathrm{Li}$ nanostructure ${ }^{38}$ or to improve the stability of the SEI layer ${ }^{39}$ appear more suitable than conventional mechanical suppression or formation of protective passivation layers. ${ }^{40}$

\section{CONCLUSIONS}

Our work represents a major step forward in understanding the evolution of electrochemically stripped/plated LmSs and may speed up the process of introducing Li into commercial lithium metal batteries (LMBs) with enhanced performance. On the one hand, ground breaking electrolytes are highly needed to facilitate the nascent electrochemically deposited LmSs to plate smoothly onto the Li bulk electrode instead of forming a highly resistive PLI. On the other hand, it is also worth investigating electrochemical $\mathrm{Li}$ stripping to enable a more uniform accessibility of $\mathrm{Li}$ ions from not only the initial bulk Li electrode but also from the electrochemically plated LmS. In addition, engineering a sophisticated architecture of the Li electrode will be technologically crucial. Finally, separators with high ion conductivities and improved rigidities are highly desirable.

\section{METHODS}

Materials. Lithium, carbon black, Polyvinylidene difluoride (PVDF) binder and Celgard separator were purchased from MTI Cor. USA. Silicon (Si) was received from Elkem AS, Norway. Electrolyte $1 \mathrm{M} \mathrm{LiPF}_{6}$ in a volume-ratio mixture (1:1) of ethylene carbonate (EC) and ethyl methyl carbonate (EMC) was purchased from Sigma Aldrich as well as N-methyl pyrrolidone solvent (NMP). The housing of the proof-of-concept beamline battery is made of polyamide-imide (Torlon) provided by Drake Plastics Europe.

Battery Preparation. The lithium electrodes in both Li-Li symmetrical cells and Li-Si half cells were punched out ( $2.5 \mathrm{~mm}$ diameter) from a lithium plate $(1 \mathrm{~mm}$ thick). The $\mathrm{Si}$ composite electrode was made of electrode slurries containing weight ratios of Si:carbon black:binder of 70:20:10 in NMP. Two kinds of Si/C electrodes were prepared: electrodes containing i) Si particles with a diameter of $\sim 20 \mu \mathrm{m}$ (for Li-Si-1 and Li-Si-3); ii) Si particles with diameters of $\sim 20, \sim 80$ and $\sim 150 \mu \mathrm{m}$ in a weight ratio of 1:1:1 (for Li-Si-2). The slurry was cast onto copper foil. To remove the NMP, the cast copper foils were dried in an oven at $60{ }^{\circ} \mathrm{C}$ for $12 \mathrm{~h}$. After drying, the composite electrode was diced into small blocks of around $1.7 \mathrm{~mm} \times 1.7 \mathrm{~mm} \times 0.2 \mathrm{~mm}$ size with a razor blade. For the Li-Si half cells, Li was used as the anode and a $\mathrm{Si} / \mathrm{C}$ electrode as the cathode. For the Li-Li symmetrical cells, Li was used both as anode and cathode. All cells (6 in total, $3 \mathrm{Li}-\mathrm{Li}$ cells from $\mathrm{Li}-\mathrm{Li}-1$ to $\mathrm{Li}-\mathrm{Li}-3,3 \mathrm{Li}-\mathrm{Si}$ cells with Li-Si-1 to Li-Si-3) were assembled in an argon-filled glovebox with humidity and oxygen levels below $0.1 \mathrm{ppm}$. The polymer separator (3.5 mm in diameter and 25 $\mu \mathrm{m}$ thick) was placed between the Li-Li electrode (in Li-Li cells) and the Li-Si electrode (in Li-Si cells). All cells were assembled manually without exerting force. Finally, both cells were filled with the liquid electrolyte and were sealed off before taking out of glovebox.

Electrochemical Measurement. Galvanostatic charge and discharge of all the batteries was carried out using an IviumStat from Ivium Technologies, Netherlerlands. The cells were subjected to different current densities for discharge and charge. It has to be noted that the electrochemically "stripping and/or plating" is from the point view of Li negative electrode in Li-Li cell system. The cycling procedure for each cell is detailed in SI.

Setting of Tomography Measurement. Synchrotron X-ray tomography was carried out at the BAMline at BESSY II of the Helmholtz-Centre Berlin, Germany. The synchrotron beam 
was monochromatized to $20 \mathrm{keV}$ using a double multilayer monochromator with an energy resolution of about $1.5 \%$. The detector system comprised a $60-\mu \mathrm{m}$ thick $\mathrm{CdWO}_{4}$ scintillator, a microscopic optic and a pco4000 camera equipped with a $4008 \times 2672$ pixels CCD chip that is kept out of the direct beam by using a mirror. For tomography measurements of all cells, 2200 projections within a $180^{\circ}$ battery rotation were recorded. For the Li-Li symmetrical cells, the exposure time was $2.5 \mathrm{~s}$, for Li-Si half cells $3 \mathrm{~s}$.

Data Processing. The raw tomography data was filtered, normalized and reconstructed using code programmed in IDL 8.2. Three-dimensional segmentations of the separators were made using a grid of manually marked points that were fitted with a biharmonic equation using MATLAB. For the segmentation of LmSs and voids, the statistical region merger tool implemented in Fiji ${ }^{41}$ was used followed by manual removal of the bulk lithium background. For the three-dimensional presentations in Figure 2 and 3, the segmentation of all materials except the $\mathrm{Li}$ bulk ( $\mathrm{Li}$ bulk set to 0 , all other materials to 1) was used as a mask for the according X-ray dataset in order to be able to show LmSs and voids without the Li bulk in the background. Two-dimensional segmentations were made by first manually segmenting the separator, followed by a statistical region merging and manualvia removal of the Li bulk background. The tomograms shown in the first column of Figure 2 are absorption contrast tomograms while the second column displays the averages of the according absorption and phase contrast tomograms. All remaining tomograms show absorption contrast only.

Supporting Information Available. Detailed cycling procedures for all cells and Figure S1S8 are included in the supporting information files. Videos showing the 3D nature of the electrode are also included. These materials are available free of charge via the internet at http://pubs.acs.org.

Remark concerning the experimental program. The effect of the pressure subjected to both cells and the electrode morphology on LmS formation was currently not the scope of this paper. Moreover, we have used a relatively small current and long cycle time to induce as much as possible $\mathrm{Li}$ dissolution and deposition. The effect of different $\mathrm{C}$ rates on the morphology of Li dissolution and deposition was not investigated. Nevertheless, these important parameters are worth to be studied in the future.

A quantitative analysis of the amount of lithium that has been dissolved from the Li anode and the amount of LmS that has been deposited onto the surface of the Li cathode (alloyed with the $\mathrm{Si} / \mathrm{C}$ cathode) has presently not been conducted mainly because the currently used $\mathrm{Li}$ electrode (2.5 mm diameter) is bigger than the field of view (FoV), which is $1.7 \mathrm{~mm}$ wide. The current that passes through the chemical workstation may give rise to dissolution or deposition of $\mathrm{Li}$ outside the FoV. Considering this, the present research focuses on the qualitative analysis of the morphological evolution of LmSs in Li electrodes. However, cells assembled with Li electrodes smaller than the FoV could be measured in the future and analysed quantitatively.

Acknowledgements. We thank Dr. Heinrich Riesemeier, the beamline scientist at BESSY II, for his valuable assistance and engineer Norbert Beck for fabricating the beamline battery. We also thank Elkem AS for providing us with the Si particles. This work is sponsored by the Helmholtz Association and the China Scholarship Council.

Author Contributions. Fu Sun, Henning Markötter and Ingo Manke designed the cell. Fu Sun, Henning Markötter and Dong Zhou assembled and tested the cells. André Hilger and Henning Markötter aided in synchrotron characterizations and the discussion of results. Lukas Zielke, André Hilger, Roland Zengerle, Riko Moroni and Simon Thiele reconstructed the data and prepared the figures. Fu Sun, Henning Markötter, Ingo Manke and John Banhart 
contributed to the data interpretation and the discussion of the results. Fu Sun, Henning Markötter, Ingo Manke and John Banhart composed the manuscript. Ingo Manke and John Banhart directed the work.

Additional information. Supplementary information is available. 


\section{REFERENCES AND NOTES}

1. Withers, P. J. Mechanical Failure: Imaging Cracks in Hostile Regimes. Nat Mater 2013, 12, 7-9.

2. Verde, M. G.; Baggetto, L.; Balke, N.; Veith, G. M.; Seo, J. K.; Wang, Z.; Meng, Y. S. Elucidating the Phase Transformation of Li4ti5o12 Lithiation at the Nanoscale. ACS Nano 2016, 10, 4312-4321.

3. Leenheer, A. J.; Jungjohann, K. L.; Zavadil, K. R.; Harris, C. T. Phase Boundary Propagation in Li-Alloying Battery Electrodes Revealed by Liquid-Cell Transmission Electron Microscopy. ACS Nano 2016, 10, 5670-5678.

4. Robert, D.; Douillard, T.; Boulineau, A.; Brunetti, G.; Nowakowski, P.; Venet, D.; BayleGuillemaud, P.; Cayron, C. Multiscale Phase Mapping of Lifepo4-Based Electrodes by Transmission Electron Microscopy and Electron Forward Scattering Diffraction. ACS Nano 2013, 7, 10887-10894.

5. Park, G. O.; Yoon, J.; Park, E.; Park, S. B.; Kim, H.; Kim, K. H.; Jin, X.; Shin, T. J.; Kim, H.; Yoon, W.-S.; Kim, J. M. In Operando Monitoring of the Pore Dynamics in Ordered Mesoporous Electrode Materials by Small Angle X-Ray Scattering. ACS Nano 2015, 9, 5470-5477.

6. Shrestha, B. R.; Baimpos, T.; Raman, S.; Valtiner, M. Angstrom-Resolved Real-Time Dissection of Electrochemically Active Noble Metal Interfaces. ACS Nano 2014, 8, 59795987.

7. Steiger, J.; Kramer, D.; Mönig, R. Mechanisms of Dendritic Growth Investigated by in Situ Light Microscopy During Electrodeposition and Dissolution of Lithium. J. Power Sources 2014, 261, 112-119.

8. Cohen, Y. S.; Cohen, Y.; Aurbach, D. Micromorphological Studies of Lithium Electrodes in Alkyl Carbonate Solutions Using in Situ Atomic Force Microscopy. J. Phys. Chem. B 2000, 104, 12282-12291.

9. Dollé, M.; Sannier, L.; Beaudoin, B.; Trentin, M.; Tarascon, J.-M. Live Scanning Electron Microscope Observations of Dendritic Growth in Lithium/Polymer Cells. Electrochem. Solid-State Lett. 2002, 5, A286-A289.

10. Leenheer, A. J.; Jungjohann, K. L.; Zavadil, K. R.; Sullivan, J. P.; Harris, C. T. Lithium Electrodeposition Dynamics in Aprotic Electrolyte Observed in Situ Via Transmission Electron Microscopy. ACS Nano 2015, 9, 4379-4389.

11. Bhattacharyya, R.; Key, B.; Chen, H.; Best, A. S.; Hollenkamp, A. F.; Grey, C. P. In Situ Nmr Observation of the Formation of Metallic Lithium Microstructures in Lithium Batteries. Nat Mater 2010, 9, 504-510.

12. Chandrashekar, S.; Trease, N. M.; Chang, H. J.; Du, L.-S.; Grey, C. P.; Jerschow, A. 7li Mri of Li Batteries Reveals Location of Microstructural Lithium. Nat Mater 2012, 11, 311-315.

13. Xu, W.; Wang, J.; Ding, F.; Chen, X.; Nasybulin, E.; Zhang, Y.; Zhang, J.-G. Lithium Metal Anodes for Rechargeable Batteries. Energy Environ. Sci. 2014, 7, 513-537.

14. Liang, Z.; Zheng, G.; Liu, C.; Liu, N.; Li, W.; Yan, K.; Yao, H.; Hsu, P.-C.; Chu, S.; Cui, Y. Polymer Nanofiber-Guided Uniform Lithium Deposition for Battery Electrodes. Nano Lett. 2015, 15, 2910-2916.

15. Ji, X.; Liu, D.-Y.; Prendiville, D. G.; Zhang, Y.; Liu, X.; Stucky, G. D. Spatially Heterogeneous Carbon-Fiber Papers as Surface Dendrite-Free Current Collectors for Lithium Deposition. Nano Today 2012, 7, 10-20.

16. Stark, J. K.; Ding, Y.; Kohl, P. A. Dendrite-Free Electrodeposition and Reoxidation of Lithium-Sodium Alloy for Metal-Anode Battery. J. Electrochem. Soc. 2011, 158, A1100A1105. 
17. Orsini, F.; Du Pasquier, A.; Beaudoin, B.; Tarascon, J. M.; Trentin, M.; Langenhuizen, N.; De Beer, E.; Notten, P. In Situ Scanning Electron Microscopy (Sem) Observation of Interfaces within Plastic Lithium Batteries. J. Power Sources 1998, 76, 19-29.

18. Eastwood, D. S.; Bayley, P. M.; Chang, H. J.; Taiwo, O. O.; Vila-Comamala, J.; Brett, D. J. L.; Rau, C.; Withers, P. J.; Shearing, P. R.; Grey, C. P.; Lee, P. D. Three-Dimensional Characterization of Electrodeposited Lithium Microstructures Using Synchrotron X-Ray Phase Contrast Imaging. Chem. Commun. 2015, 51, 266-268.

19. Harry, K. J.; Hallinan, D. T.; Parkinson, D. Y.; MacDowell, A. A.; Balsara, N. P. Detection of Subsurface Structures Underneath Dendrites Formed on Cycled Lithium Metal Electrodes. Nat Mater 2014, 13, 69-73.

20. Lu, D.; Shao, Y.; Lozano, T.; Bennett, W. D.; Graff, G. L.; Polzin, B.; Zhang, J.; Engelhard, M. H.; Saenz, N. T.; Henderson, W. A.; Bhattacharya, P.; Liu, J.; Xiao, J. Failure Mechanism for Fast-Charged Lithium Metal Batteries with Liquid Electrolytes. Adv. Energy Mater. 2015, 5, 1400993-1401000.

21. Manke, I.; Banhart, J.; Haibel, A.; Rack, A.; Zabler, S.; Kardjilov, N.; Hilger, A.; Melzer, A.; Riesemeier, H. In Situ Investigation of the Discharge of Alkaline Zn-Mno2 Batteries with Synchrotron X-Ray and Neutron Tomographies. Appl. Phys. Lett. 2007, 90, 214102214105.

22. Sun, F.; Markötter, H.; Zhou, D.; Alrwashdeh, S. S. S.; Hilger, A.; Kardjilov, N.; Manke, I.; Banhart, J. In Situ Radiographic Investigation of (De)Lithiation Mechanisms in a TinElectrode Lithium-Ion Battery. ChemSusChem 2016, 9, 946-950.

23. Sun, F.; Markötter, H.; Manke, I.; Hilger, A.; Kardjilov, N.; Banhart, J. ThreeDimensional Visualization of Gas Evolution and Channel Formation inside a Lithium-Ion Battery. Acs Appl. Mater. Interfaces 2016, 8, 7156-7164.

24. Sun, F.; Markötter, H.; Dong, K.; Manke, I.; Hilger, A.; Kardjilov, N.; Banhart, J. Investigation of Failure Mechanisms in Silicon Based Half Cells During the First Cycle by Micro X-Ray Tomography and Radiography. J. Power Sources 2016, 321, 174-184.

25. Zielke, L.; Barchasz, C.; Waluś, S.; Alloin, F.; Leprêtre, J. C.; Spettl, A.; Schmidt, V.; Hilger, A.; Manke, I.; Banhart, J.; Zengerle, R.; Thiele, S. Degradation of Li/S Battery Electrodes on 3d Current Collectors Studied Using X-Ray Phase Contrast Tomography. Sci. Rep. 2015, 5, 10921-10933.

26. Zielke, L.; Sun, F.; Markötter, H.; Hilger, A.; Moroni, R.; Zengerle, R.; Thiele, S.; Banhart, J.; Manke, I. Synchrotron X-Ray Tomographic Study of a Silicon Electrode before and after Discharge and the Effect of Cavities on Particle Fracturing. ChemElectroChem 2016, 3, 1170-1177.

27. Zielke, L.; Hutzenlaub, T.; Wheeler, D. R.; Chao, C.-W.; Manke, I.; Hilger, A.; Paust, N.; Zengerle, R.; Thiele, S. Three-Phase Multiscale Modeling of a Licoo2 Cathode: Combining the Advantages of Fib-Sem Imaging and X-Ray Tomography. Adv. Energy Mater. 2015, 5, 1401612-1401620.

28. Eastwood, D. S.; Bradley, R. S.; Tariq, F.; Cooper, S. J.; Taiwo, O. O.; Gelb, J.; Merkle, A.; Brett, D. J. L.; Brandon, N. P.; Withers, P. J.; Lee, P. D.; Shearing, P. R. The Application of Phase Contrast X-Ray Techniques for Imaging Li-Ion Battery Electrodes. Nucl. Instrum. Meth. B 2014, 324, 118-123.

29. Li, W.; Zheng, H.; Chu, G.; Luo, F.; Zheng, J.; Xiao, D.; Li, X.; Gu, L.; Li, H.; Wei, X.; Chen, Q.; Chen, L. Effect of Electrochemical Dissolution and Deposition Order on Lithium Dendrite Formation: A Top View Investigation. Faraday. Discuss. 2014, 176, 109-124.

30. Arakawa, M.; Tobishima, S.-i.; Nemoto, Y.; Ichimura, M.; Yamaki, J.-i. Lithium Electrode Cycleability and Morphology Dependence on Current Density. J. Power Sources 1993, 43, 27-35. 
31. Grande, L.; von Zamory, J.; Koch, S. L.; Kalhoff, J.; Paillard, E.; Passerini, S. Homogeneous Lithium Electrodeposition with Pyrrolidinium-Based Ionic Liquid Electrolytes. Acs Appl. Mater. Interfaces 2015, 7, 5950-5958.

32. Love, C. T. Thermomechanical Analysis and Durability of Commercial Micro-Porous Polymer Li-Ion Battery Separators. J. Power Sources 2011, 196, 2905-2912.

33. Gonzalez, J.; Sun, K.; Huang, M.; Lambros, J.; Dillon, S.; Chasiotis, I. Three Dimensional Studies of Particle Failure in Silicon Based Composite Electrodes for Lithium Ion Batteries. J. Power Sources 2014, 269, 334-343.

34. Jana, A.; Ely, D. R.; García, R. E. Dendrite-Separator Interactions in Lithium-Based Batteries. J. Power Sources 2015, 275, 912-921.

35. Ryou, M.-H.; Lee, Y. M.; Lee, Y.; Winter, M.; Bieker, P. Mechanical Surface Modification of Lithium Metal: Towards Improved Li Metal Anode Performance by Directed Li Plating. Adv. Funct. Mater. 2015, 25, 834-841.

36. Heine, J.; Krüger, S.; Hartnig, C.; Wietelmann, U.; Winter, M.; Bieker, P. Coated Lithium Powder (Clip) Electrodes for Lithium-Metal Batteries. Adv. Energy Mater. 2014, 4, 1300815-1300822.

37. Yan, K.; Lu, Z.; Lee, H.-W.; Xiong, F.; Hsu, P.-C.; Li, Y.; Zhao, J.; Chu, S.; Cui, Y. Selective Deposition and Stable Encapsulation of Lithium through Heterogeneous Seeded Growth. Nat. Energy. 2016, 1, 16010-16018.

38. Zhang, Y.; Qian, J.; Xu, W.; Russell, S. M.; Chen, X.; Nasybulin, E.; Bhattacharya, P.; Engelhard, M. H.; Mei, D.; Cao, R.; Ding, F.; Cresce, A. V.; Xu, K.; Zhang, J.-G. Dendrite-Free Lithium Deposition with Self-Aligned Nanorod Structure. Nano Lett. 2014, 14, 6889-6896.

39. Zheng, J.; Yan, P.; Mei, D.; Engelhard, M. H.; Cartmell, S. S.; Polzin, B. J.; Wang, C.; Zhang, J.-G.; Xu, W. Highly Stable Operation of Lithium Metal Batteries Enabled by the Formation of a Transient High-Concentration Electrolyte Layer. Adv. Energy Mater. 2016, 6, 1502151-1502161.

40. Zheng, G.; Lee, S. W.; Liang, Z.; Lee, H.-W.; Yan, K.; Yao, H.; Wang, H.; Li, W.; Chu, S.; Cui, Y. Interconnected Hollow Carbon Nanospheres for Stable Lithium Metal Anodes. Nat Nano 2014, 9, 618-623.

41. Abramoff, M. D.; Magalhães, P. J.; Ram, S. J. Image Processing with Imagej. Biophotonics international 2004, 11, 36-42. 